\title{
Tos17 rice element: incomplete but effective
}

\author{
Francois Sabot
}

\begin{abstract}
Background: Tos 17 was the first LTR retrotransposon (Copia) described as active in cultivated rice, and is present in two copies in the genome of the sequenced Nipponbare variety. Only the chromosome 7 copy is active and able to retrotranspose, at least during in vitro culture, and this ability was widely used in insertional mutagenesis assays.

Results: Here the structure of the active Tos 17 was thoroughly annotated using a set of bioinformatic analyses.

Conclusions: Unexpectedly, Tos 17 appears to be a non-autonomous LTR retrotransposon, lacking the gag sequence and thus unable to transpose by itself.
\end{abstract}

\section{Background}

The long terminal repeats (LTR) retrotransposon life cycle involves a cytosolic reverse-transcription step within a multiproteic core called virus-like particle (VLP), formed by the polymerization of the Group-specific antigen (GAG) proteins, normally encoded in the element itself; for a recent review, see [1]. This GAG protein classically harbors three domains, from external to internal:

1) the matrix domain (MA), for membrane targeting and capsid assembly;

2) the capsid hydrophobic region (CA) and the most conserved part of GAG, in charge of polymerization, and the

3) nucleocapsid (NC), targeting the specific mRNA through the PSI region [1].

In addition, a $\mathrm{CCHC}$ zinc-finger motif is located at the $\mathrm{C}$-terminus of the protein, single or twice repeated (or even thrice), and is in charge of the protein-nucleic acid interactions [1]. This protein is theoretically specific of its own RNA, and is an essential and mandatory component of the retrotransposition of LTR retrotransposons. A second open reading frame (ORF), pol, encodes the reverse transcriptase-RNaseH (RT-RNaseH), which drives the synthesis of a double-stranded cDNA from two RNA matrices and the integrase (INT) which allows the insertion of the new cDNA copy. However, in some cases,

Correspondence: francois.sabot@ird.fr

UMR DIADE IRD/UM2, 911 Avenue Agropolis BP64503, F-34394 Montpellier Cedex 5, France

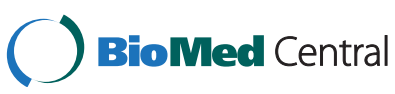

some non-autonomous elements have been shown capable of hijacking the GAG from other elements [2].

In cultivated Asian rice (Oryza sativa L.), LTR retrotransposons compose at least $20 \%$ of the genome (MSUv7.0 reference genome [3], http://rice.plantbiology.msu.edu/ index.shtml). The Copia Tos17 element (for Transposon of Oryza sativa 17) was the first identified as active [4] and able to transpose in this genome. Moreover, Tos17 seems to be the most transpositionally competent one in regenerated plants [5].

Two almost identical genomic copies of Tos 17 reside in the reference genome (on chromosomes 7 and 10; Figure 1). Only the chromosome-7 copy is transpositionally active (during in vitro culture at least), whereas the other, located on chromosome 10, is inactive, heavily methylated and contains several stop codons and indels in its predicted coding region [6]. This last copy can, however, be reactivated (transcriptionally) in methylation-defective mutants [6]. In the whole Oryza genus, the copy number as well as the location of active copies (if there are any) may differ [7].

The Tos 17 activation during in vitro culture was widely used in mutagenesis assays, which allowed reverse genetics analyses through the generation of insertional mutants without transformation [8-10]. In the present study, a detailed functional analysis of Tos17 was performed, showing that both genomic Tos 17 copies lack a gag ORF, making Tos17 a non-autonomous element requiring an active one in order to ensure its transposition.

\section{Results and discussion}

The two Tos17 genomic copies were extracted from their respective location in the rice MSUv7.0 genome, 


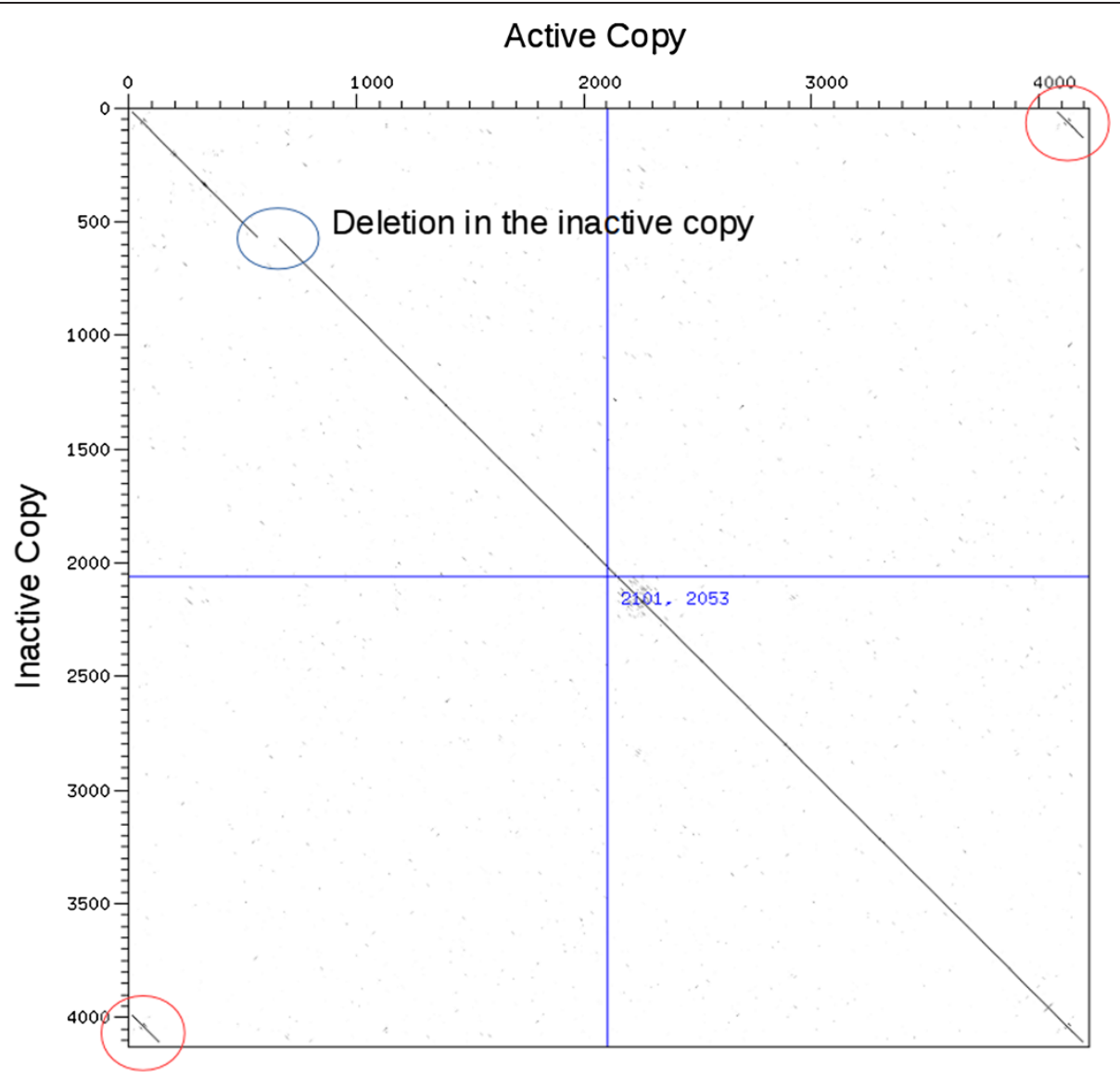

Figure 1 Dotter alignment of the two Tos 17 genomic copies. Horizontally, the copy from chromosome 7 (active copy); vertically, the copy from chromosome 10 (inactive copy). The difference in transcriptional/transpositional activity is due to small mismatches, forming stop-codons and frameshifting, and to a larger deletion in the inactive copy (blue circle). The long terminal repeats (LTR) repeated sequences are circled in red.

and manually annotated using a series of basic local alignment search tool (BLAST), ProSite and Protein families (Pfam) analyses. A predicted long ORF (from position 659 to 3835, Figure 2A; annotated as the gag-pol ORF [4]) of 1,058 residues can be detected on the active copy (chromosome 7), whereas no apparent ORFs (that is, more than 100 residues starting with Met) exist on the inactive copy. On this long ORF, INT (gag_pre-integrase and rve) and RT (RVT_2) Pfam-A motifs can be easily identified (see Table 1), which suggests that this ORF is the polyprotein (POL) one. However, none of the truly GAG-related motifs, such as $\mathrm{CCHC}$ zinc-finger (18 residues) or the UBN2 group (100 to 150 residues), could be identified, and the first confidently identified motif related to the INT (and thus to the pol ORF) in the Pfam database starts at residue 79 (Figure 2A; gag_pre-integrase motif) of this ORF (base 757 of the internal sequence).

The Pfam analysis was performed on the largest Tos17 ORF.

This ORF was then compared to ORFs from those of the active Copia elements, RIRE1 from Oryza. australiensis [11,12] [BAA22288; EMBL/GB] (Figure 2B), and Houba

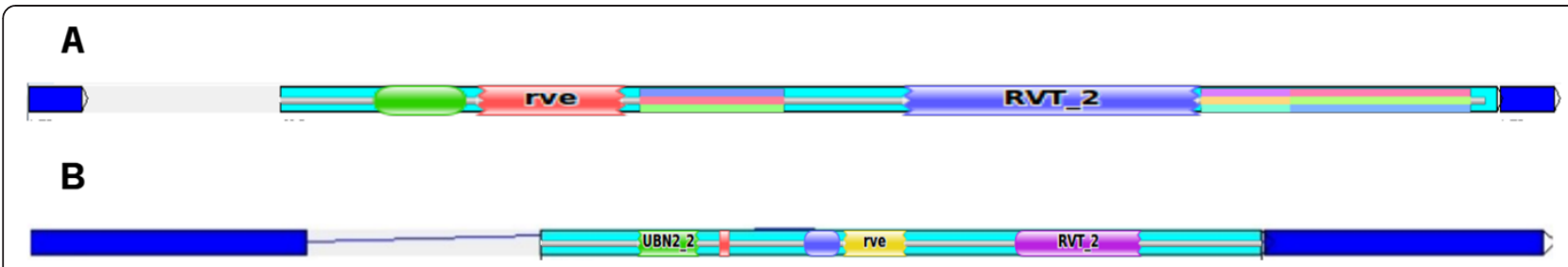

Figure 2 Annotation of Tos17 and RIRE1. Long terminal repeats (LTR) are symbolized in dark blue and the open reading frame (ORF) in light blue. (A) Tos 17 annotation. The gag_pre-integrase (green), rve (red) and RVT_2 (blue) motif positions are reported on the ORF. (B) RIRE1 annotation. The UBN2_2 (green), Zf-CCHC (red), gag_pre-integrase (blue), rve (yellow) and RVT_2 (purple) motif positions are reported on the ORF. 
Table 1 Tos17 Open reading frame (ORF)2 Pfam motifs

\begin{tabular}{llll}
\hline Motif & Start & Stop & e-value \\
\hline gag_pre-integrase PF13976 & 79 & 153 & $6.50 \mathrm{e}-016$ \\
rve PF00665 & 164 & 284 & $3.60 \mathrm{e}-026$ \\
RVT_2 PF07727 & 519 & 762 & $1.10 \mathrm{e}-095$ \\
\hline
\end{tabular}

from O. sativa (known to be one of the most recently retrotransposed Copia in rice; [13]). As shown on Figure 3, the ORFs aligned on the whole POL part the elements that are compared two by two; the Tos 17 ORF, however, lacked the GAG region, while the ORFs from RIRE1 and Houba are also aligned on the GAG part. No GAG-related region can be detected on the whole Tos17 genomic sequences in BLASTX against $n r$ and protein databases (data not shown). Various $t B L A S T n$ (protein query versus nucleic database) analyses against the rice EST databases from NCBI were performed, and no ESTs resembling a larger ORF than the ones known were detected. Finally, no other Tos17 gag-like sequence can be amplified in PCR on the NipponBare genomic DNA (data not shown).

RT-sequence phylogenetic analysis showed that only RN304 and Lullaby are closely related to Tos17 [14]. Interestingly, RN304, the closest element to Tos 17, is itself also a non-autonomous element also lacking the gag sequence, similar to Tos17, but no information about its transpositional activity is available. The closest complete element to Tos17 (that is, one that harbors a complete gag-pol ORF) is the Lullaby element, recently shown as transitionally active in only some of the regenerated lines in which its expression was detected [14]. Lullaby is a 5'142-long element, and has two copies in the Nipponbare genome, on chromosomes 6 and 9, with only the chromosome-6 copy active [14]. The DNA similarity between Tos17 and Lullaby is $57 \%$ at the DNA level (whole element sequence), and 64\% at the protein level (gag-pol region sequence). At the DNA level, the similarity is limited to the internal sequence, whereas at the protein level the two POL sequences aligned well. Moreover, the primer binding site (PBS) region, located immediately after the $5^{\prime}$ LTR, and involved in RNA-GAG recognition [1], is almost identical between the two elements $\left(5^{\prime}\right.$ TGGTATCAGAGC(a/t)A(t/-)GGT-3'), starting at positions 126 and 139 for Lullaby and Tos17 respectively. However, no common INT signal (at the 3'-end of the 3' LTR [1]) is shared between Lullaby and Tos17, highlighting the use of Lullaby GAG by Tos 17 only.

Tos 17, the most active LTR retrotransposon in cultivated rice, and the most commonly used element as an insertional mutation tool [8], is thus a non-autonomous element, because no gag sequence exists in the Oryza sativa genome, even if Tos 17 is able to retrotranspose in this species. The simplest explanation is that Tos 17 is coupled with an active LTR retrotransposon for its mobility, and that the former is able to use the gag (and VLP) from the latter. Such hitchhiking implies a structural (same GAG-recognition signals) as well as translational (same time of expression) relationship between Tos17 and its autonomous partner. This association is probably a long-term association, as the structural annotation of the Tos17 elements (Figure 2A) reveals a complete removal of the gag region, without any identifiable remnants, but without damaging any other structural features of the element (LTR, PBS or polypurine tract (PPT)). Indeed, such clean elimination might have occurred during Tos 17 evolution, with only elements within this correct deletion selected (able to be correctly expressed and mobilized by its partner), as no other Tos17-like element with gag remnants has been detected.

The use of Tos17 as an insertional tool for reverse genetics is not affected by this non-autonomous state, as long as requested functional and complementation analyses are performed to validate or invalidate the insertion as the real cause of the observed phenotype.

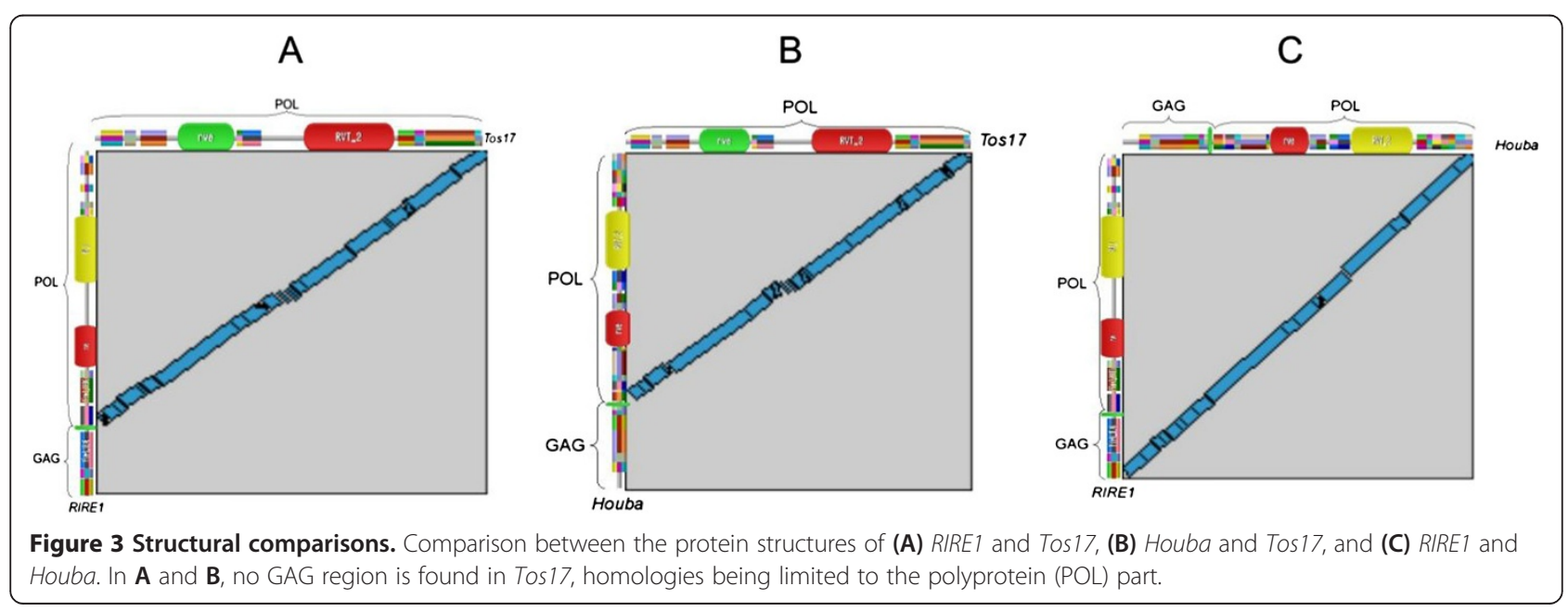


The fact that Tos 17 is not able to retrotranspose by itself may help to explain the high rate (almost 90\%) of morpho-physiological variations untagged by Tos17 (or the transferred T-DNA) observed among regenerated lines ([9]; M Lorieux, unpublished data; B Hsingh, personal communication), which is probably also due to transposition of other elements, as shown previously [5].

\section{Conclusion}

Analyses, such as the one described here, highlight the need for a better knowledge of transposable elements (TEs), in order to ensure a better understanding of their effects upon the host genome. In particular, it may be of interest to further study the details of the relationships between the non-autonomous elements and their autonomous counterparts, because existing data suggest that the former are more active than the latter, as shown for BARE2 and Tos17.

\section{Methods}

The nucleotidic sequences from genomic copies of each element were launched in Artemis [15], and the ORFs longer than 100 residues were automatically extracted from the element sequences. The ORFs were then scanned online using a combination of Pfam, ProSite and BLASTp analyses [16] with standard parameters. The results were then reported on Artemis, in order to manually reconstruct the complete structure of each element. The LTRs were identified using Dotter [17], and the PBS and PPT were manually determined. The comparison between putative GAG-POL sequences was performed using the Align2Sequence graphical tool from the NCBI, through a BLASTp analysis, for a better presentation. The identity/similarity levels were calculated using the Stretcher program from the EMBOSS suite.

\section{Abbreviations}

BLAST: basic local alignment search tool; GAG: group-specific antigene; INT: integrase; LTR: long terminal repeats; ORF: open reading frame; PBS: primer binding site; POL: Polyprotein; PPT: Polypurine tract; TE: transposable element;

Tos: transposon of Oryza sativa; VLP: virus-like particle.

\section{Competing interests}

The author declares having no competing interests.

\section{Acknowledgements}

The author thanks Cristian Chaparro and Benoit Piegu for their comments on the analyses, and Dr Timothy Tranberger for his help with English corrections.

Received: 22 November 2013 Accepted: 11 March 2014

Published: 1 April 2014

\section{References}

1. Sabot F, Schulman AH: Parasitism and the retrotransposon life cycle in plants: a hitchhiker's guide to the genome. Heredity 2006, 97:381-388.

2. Tanskanen JA, Sabot F, Vicient C, Schulman AH: Life without GAG: the BARE-2 retrotransposon as a parasite's parasite. Gene 2007, 390:166-174.

3. Kawahara Y, de la Bastide M, Hamilton JP, Kanamori H, McCombie WR, Ouyang S, Schwartz DC, Tanaka T, Wu J, Zhou S, Childs KL, Davidson RM, Lin H, Quesada-Ocampo L, Vaillancourt B, Sakai H, Lee SS, Kim J, Numa H,
Itoh T, Buell CR, Matsumoto T: Improvement of the Oryza sativa Nipponbare reference genome using next generation sequence and optical map data. Rice 2013, 6:4.

4. Hirochika H, Sugimoto K, Otsuki Y, Tsugawa H, Kanda M: Retrotransposons of rice involved in mutations induced by tissue culture. Proc Natl Acad Sci USA 1996, 93:7783-7788.

5. Sabot F, Picault N, ElBaidouri M, Llauro C, Chaparro C, Piegu B, Roulin A, Guiderdoni E, Delabastide M, McCOMBIE R, Panaud O: Transpositional landscape of the rice genome revealed by paired-end mapping of high-throughput re-sequencing data. Plant J 2011, 66:241-246.

6. Hirochika H: Contribution of the Tos 17 retrotransposon to rice functional genomics. Curr Opin Plant Biol 2001, 4:118-122.

7. Petit J, Bourgeois E, Stenger W, Bès M, Droc G, Meynard D, Courtois B, Ghesquière A, Sabot F, Panaud O, Guiderdoni E: Diversity of the Ty-1 copia retrotransposon Tos 17 in rice (Oryza sativa L.) and the AA genome of the Oryza genus. Mol Genet Genom 2009, 282:633-652.

8. Hirochika H, Guiderdoni E, An G, Hsing Y-I, Eun MY, Han C-D, Upadhyaya N, Ramachandran S, Zhang Q, Pereira A, Sundaresan V, Leung H: Rice mutant resources for gene discovery. Plant Mol Biol 2004, 54:325-334.

9. Miyao A, Iwasaki Y, Kitano H, Itoh J-I, Maekawa M, Murata K, Yatou O, Nagato Y, Hirochika H: A large-scale collection of phenotypic data describing an insertional mutant population to facilitate functional analysis of rice genes. Plant Mol Biol 2007, 63:625-635.

10. Piffanelli $P$, Droc $G$, Mieulet $D$, Lanau N, Bès $M$, Bourgeois $E$, Rouvière $C$, Gavory F, Cruaud C, Ghesquière A, Guiderdoni E: Large-scale characterization of Tos 17 insertion sites in a rice T-DNA mutant library. Plant Mol Biol 2007, 65:587-601.

11. Noma $\mathrm{K}$, Nakajima $\mathrm{R}$, Ohtsubo $\mathrm{H}$, Ohtsubo E: RIRE1, a retrotransposon from wild rice Oryza australiensis. Genes Genet Syst 1997, 72:131-140.

12. Piegu B, Guyot R, Picault N, Roulin A, Sanyal A, Saniyal A, Kim H, Collura K, Brar DS, Jackson S, Wing RA, Panaud O: Doubling genome size without polyploidization: dynamics of retrotransposition-driven genomic expansions in Oryza australiensis, a wild relative of rice. Genome Res 2006, 16:1262-1269.

13. Vitte C, Ishii T, Lamy F, Brar D, Panaud O: Genomic paleontology provides evidence for two distinct origins of Asian rice (Oryza sativa L.). Mol Genet Genom 2004, 272:504-511.

14. Picault N, Chaparro C, Piegu B, Stenger W, Formey D, Llauro C, Descombin J, Sabot F, Lasserre E, Meynard D, Guiderdoni E, Panaud O: Identification of an active LTR retrotransposon in rice. Plant J 2009, 58:754-765.

15. Rutherford K, Parkhill J, Crook J, Horsnell T, Barrell B, Rice P: Artemis: sequence visualization and annotation. Bioinformatics 2000, 16:944-945.

16. Altschul SF, Gish W, Miller W, Myers EW, Lipman DJ: Basic local alignment search tool. J Mol Biol 1990, 215:403-410.

17. Sonnhammer ELL, Durbin R: A dot-matrix program with dynamic threshold control suited for genomic DNA and protein sequence analysis. Gene 1996, 167:1-10.

doi:10.1186/1759-8753-5-10

Cite this article as: Sabot: Tos 17 rice element: incomplete but effective. Mobile DNA 2014 5:10.

\section{Submit your next manuscript to BioMed Central and take full advantage of:}

- Convenient online submission

- Thorough peer review

- No space constraints or color figure charges

- Immediate publication on acceptance

- Inclusion in PubMed, CAS, Scopus and Google Scholar

- Research which is freely available for redistribution 